\title{
Ocular Morbidity After Radiotherapy of Orbital and Conjunctival Lymphoma
}

\author{
E. M. BESSELL, J. M. HENK, R. A. F. WHITELOCKE and J. E. WRIGHT* \\ London
}

\begin{abstract}
Summary
One hundred and fifteen patients were treated for lymphoma of the conjunctiva and orbit between 1970 and 1984. One hundred and twelve patients received radiotherapy to the orbit, of whom 73 (65 per cent) had at least one year of follow-up by an ophthalmic surgeon. Patients with low-grade lymphomas (97) mostly received $30 \mathrm{~Gy}$ in 15 fractions to the orbit over a period of 3 weeks. Those with high-grade lymphomas (18) mostly received $40 \mathrm{~Gy}$ in 20 fractions over 4 weeks. Most were treated using anterior and lateral radiation fields to the orbit with shielding of the cornea and lens. This technique delivered a mean lens dose of $15 \mathrm{~Gy}$.

The early and late ocular and orbital morbidity in these patients was low. Eight radiation-induced cataracts developed of which only six interfered with vision. None has needed cataract surgery. No patients developed radiation retinopathy and only 5 had disorders of ocular lubrication.
\end{abstract}

Most of the previous studies of the effects of $\mathrm{X}$-rays on the eye and orbit have been concerned with cataract formation ${ }^{1,2}$ or with the effect of high doses (50 to $70 \mathrm{~Gy}$ ) administered for treatment of adjacent carcinomas of the air sinuses or nasopharynx..$^{3,4,5}$

The incidence of late ocular and orbital morbidity is high with doses of 50 to $70 \mathrm{~Gy}$; complications include disordered ocular lubrication, retinopathy and cataract. The incidence of late ocular and orbital morbidity in patients treated with lower doses in the range of 30 to $40 \mathrm{~Gy}$ has not previously been reported, because this dose level is most often used for palliative treatment, for example in patients with orbital and choroidal metastases who do not survive long enough for assess- ment. However, orbital lymphoma is treated by radiotherapy using this range of dose, and is associated with a high rate of long term survival. Accordingly, we have studied the ocular effect of radiotherapy in our patients irradiated for orbital lymphoma.

\section{Material and Methods}

Between January 1970 and December 1984, 115 patients with orbital or conjunctival non-Hodgkin's lymphoma were referred to the ophthalmic clinic at the Royal Marsden Hospital. There were 61 males and 54 females. The median age at diagnosis was 63 years (range 3 to 86 years).

\section{Tumour assessment}

The diagnosis was established by biopsy in all cases. The position and extent of the disease in the orbit 
were ascertained by clinical examination, and since 1975 were delineated by CT scanning.

The patients were examined for evidence of disseminated lymphoma, in particular looking for signs of lymphadenopathy, hepatosplenomegaly, skin and pharyngeal involvement. All patients had a full blood count and chest X-ray, most had a bone marrow biopsy, and had either bipedal lymphography or a CT scan of the abdomen and pelvis.

On the basis of the clinical findings and results of the above investigations the patients were staged according to the Ann Arbor classification. ${ }^{6}$

\section{Ophthalmic examination}

Visual acuities were measured. External examination included measurement of proptosis and assessment of eye movements. Eyes were examined before, during and after radiotherapy, using a slit lamp biomicroscope in order to assess changes in either the tear film, cornea or lens. Routine fundus examination was also carried out.

\section{Histology}

There are several different classifications of nonHodgkin's lymphoma in current use. In this report the patients are divided into 3 broad groups.

(a) High-grade malignant lymphoma, 18 patients.

(b) Low-grade malignant lymphoma, 44 patients. These were all reported as malignant tumours by at least one pathologist, or had clinical evidence of disseminated disease.

(c) Indeterminate lymphocytic lesions, 53 patients. ${ }^{7}$ These consist of lymphoid cells, but unequivocal histological features of malignancy are absent.

We regard Group C to be low-grade lymphoma, because they show the same natural history and dissemination pattern as Group B. ${ }^{8}$ There are therefore 97 patients regarded as having low-grade lymphoma, 81 had disease confined to one or both orbits (Stage IE). The tumour was bilateral in 5 patients. Sixteen had disease outside the orbit, (Stage IIE-IV); two of these had a nodular pattern, the remaining 14 were of the diffuse well-differentiated lymphocytic type.

\section{Treatment}

Only 3 patients did not receive radiotherapy to the orbit. One elderly patient with advanced immunoblastic lymphoma received no treatment, and two of the 8 patients with disseminated low-grade lymphoma (Stage IIIE or IV) were treated by chemotherapy alone. Of the 112 patients treated by radiotherapy, 3 of the 18 with high-grade lymphoma and one of the 6 with disseminated low- grade lymphoma also received chemotherapy as part of their initial management.

\section{Follow-up}

Seven patients died within one year of radiotherapy, so their late morbidity could not be assessed. Seventy-three patients ( 70 per cent) have been followed-up for at least one year either at the Ophthalmic Clinic, Royal Marsden Hospital, London (RAFW) or at Moorfields Eye Hospital, London (JEW). There were three main reasons why the assessment of late morbidity was inadequate in 32 patients:

(1) The patient was unwilling to attend a follow-up clinic mostly because of advanced age.

(2) The patient was referred to a hospital nearer home for follow-up and detailed ophthalmic examinations were not performed.

(3) The patient came from abroad and did not return sufficiently regularly for examination.

At each follow-up visit ophthalmological examination was done as described above. Fundus fluorescein angiography was performed in a randomly selected group of 10 patients who had no lens opacities.

\section{Radiotherapy technique and dose}

Patients with localised high-grade lymphoma were treated to a dose of $40 \mathrm{~Gy}$ in 20 fractions over a period of 4 weeks. Patients with low-grade lymphoma received $40 \mathrm{~Gy}$ in 20 fractions over 4 weeks until 1976, since when a dose of $30 \mathrm{~Gy}$ in 15 fractions in 3 weeks has been given. Our present policy is to treat the whole orbit using megavoltage irradiation, with a field arrangement individually planned for each patient taking into account the clinical and CT findings. The most commonly used technique consisted of a direct anterior field and a lateral field behind the lenses (Fig. 1). In most cases the cornea and lens were shielded from the anterior radiation field using a pencil-shaped lead block. Where the tumour was confined to the intraconal space lateral fields only were used.

Patients with small conjunctival lesions were generally treated with superficial X-rays (110 or $145 \mathrm{kV}$ ) and received 20 to $24 \mathrm{~Gy}$ in $10-12$ fractions in 2 weeks. Since 1975 CT scanning has been used to confirm that such lesions are limited to the conjunctiva. Fifty-nine of the 73 patients who had adequate follow-up were treated with megavoltage $\mathrm{X}$-rays using either anterior and lateral wedged fields or an anterior field alone. The lens dose was calculated for these 59 patients (64 lenses) but not for those treated with other techniques. The method of calculation of lens dose is described elsewhere. ${ }^{8}$ When the corneal shield is used the 


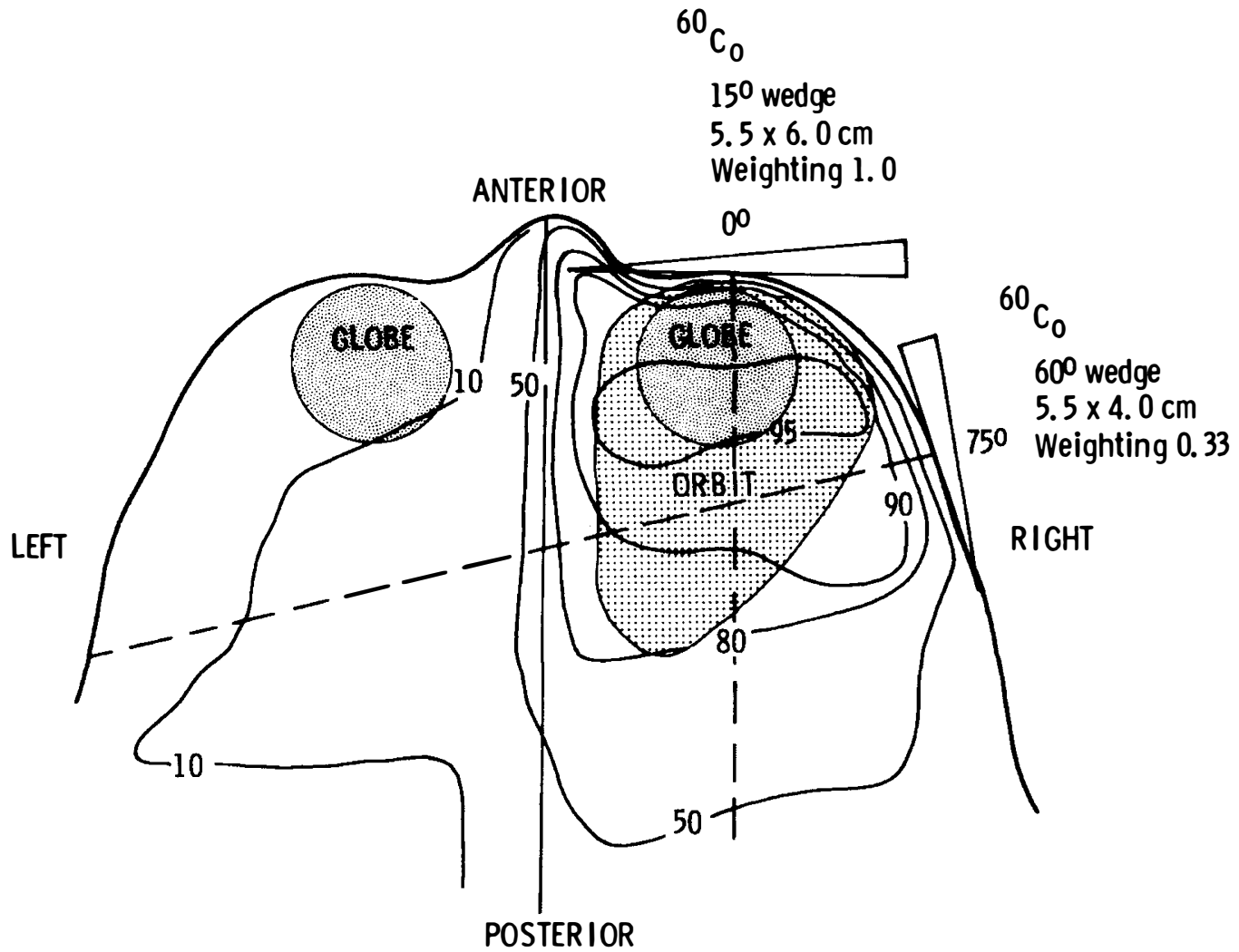

Fig. 1. Distribution of radiation dose for treatment of the right orbit with 60 co $\gamma$-rays when anterior lens shielding is not used.

absorbed dose in the lens is higher at the periphery than at the central zone. The mean lens dose calculated at a point estimated to be in the germinative zone was $15 \mathrm{~Gy}$ (see Tables I and II) with a range of 5.5 to $24.5 \mathrm{~Gy}$ (see Fig. 2) when the shield was used.

\section{Results \\ Survival}

The survival of these patients is described in detail elsewhere. ${ }^{8}$ The survival of the 97 patients with low-grade non-Hodgkin's lymphoma (Stage IE-IV) was 84 per cent at 5 years, and did not differ from the expected survival of a normal population of the same age and sex. The 5 year survival for the 18 patients with high-grade non-Hodgkin's lymphoma was 60 per cent ( 75 per cent stage IE; 40 per cent stage IIE-IV). There has been no local recurrence of lymphoma in the orbit in any of the 112 patients who received radiotherapy.

Cataract formation (see Tables I and II and Figs. 2-4)

Posterior, subcapsular lens opacities were observed in 8 patients. These were all unilateral. None of the patients who received radiotherapy to both orbits and had adequate follow-up developed a cataract. In 6 of the 8 patients there has been a reduction in visual acuity (Fig. 3) but none so far has needed cataract surgery. In 2 of these patients, cataract formation could have been enhanced by other causes. One had systemic corticosteroids, and one had severe ophthalmic herpes zoster two years after radiotherapy.

Three patients (aged 69, 73 and 73 when treated) have developed senile cortical cataracts whilst being followed-up. These lens changes were not attributed to radiotherapy 
Table I Incidence of late ocular morbidity. Cataract formation

\begin{tabular}{lcc}
\hline & $\begin{array}{c}\text { Shielded } \\
\text { lenses }\end{array}$ & $\begin{array}{c}\text { Unshielded } \\
\text { lenses }\end{array}$ \\
\hline No. of patients & 52 & 7 \\
No. of lenses & 56 & 8 \\
Orbital dose (Gy) & $20-49$ & 30 \\
Mean lens dose (Gy) & 15 & 30 \\
No. of lens opacities & 5 & 3 \\
No. with reduced visual & 3 & 3 \\
acuity & 3 & \\
\hline
\end{tabular}

Table II Incidence of late ocular morbidity. Probability of lens opacities

\begin{tabular}{lcc}
\hline & $\begin{array}{c}\text { Shielded } \\
\text { lenses }\end{array}$ & $\begin{array}{c}\text { Unshielded } \\
\text { lenses }\end{array}$ \\
\hline Mean lens dose & $15 \mathrm{~Gy}$ & $30 \mathrm{~Gy}$ \\
Risk at 5 years & $12 \%(7 \%)$ & $64 \%$ \\
Risk at 8 years & $28 \%(16 \%)$ & - \\
\hline
\end{tabular}

() numbers in brackets indicate those where lens opacities decreased visual acuity.

as they were bilateral and symmetrical, and therefore were probably age related. When the lens was shielded from the anterior radiation field and the mean lens dose was reduced to $15 \mathrm{~Gy}$ (see Tables I and II), the probability of cataract formation was 12 per cent at 5 years and 28 per cent at 8 years (Table II, Fig. 4). The probability of formation of a cataract reducing visual acuity was 7 per cent at 5 years and 16 per cent at 8 years. When the lens was not shielded and received the full radiation dose, $(30 \mathrm{~Gy})$, the probability of cataract formation with reduction of visual acuity was 64 per cent at 5 years, (Fig. 4).

No lens opacities were observed in patients receiving a lens dose of less than $10 \mathrm{~Gy}$ but the follow-up for these patients was shorter than for those receiving a higher dose and further follow-up on these patients is needed.

\section{Disordered ocular lubrication and corneal ulceration}

None of the patients receiving an orbital dose of less than $30 \mathrm{~Gy}$ complained of a 'dry eye' (see Table III). Disordered ocular lubrication was recorded as such when the patient was persistently symptomatic after the subsidence of the acute radiation reaction, and had punc- tate epithelial staining of the cornea, with normal lid-globe apposition and a reduced marginal tear meniscus. The incidence in the dose range 30 to $39 \mathrm{~Gy}$ was only 4.5 per cent (two patients). The incidence was 23 per cent (three patients) for those patients receiving 40 to 49 Gy. Only 2 patients developed a corneal ulcer and both of these eventually healed. One patient received $48 \mathrm{~Gy}$ to the orbit with 60 Co X-rays but the cornea was shielded. The other received $41 \mathrm{~Gy}$ with $250 \mathrm{kV}$ X-rays and there was no corneal shielding. Both patients also had tear film instability, which was probably the major aetiological factor in the formation of the corneal ulcer in the first patient.

\section{Retinopathy}

No radiation-induced retinopathy was observed in any treated eye. No retino-vascular changes were demonstrated in the 10 patients investigated by fundus fluorescein angiography. The retina in these 10 patients had received a radiation dose of $25-30 \mathrm{~Gy}$.

\section{Other Changes}

Mild conjunctival telangiectasia has been observed. Asteroid hyalitis was present in two of the treated eyes. This finding was thought to be coincidental.

\section{Effect of chemotherapy on morbidity}

There was no late morbidity in the three patients treated with chemotherapy alone. One of the four patients treated with chemotherapy and radiotherapy - a three year old boy with a Stage IE diffuse lymphoblastic lymphoma-developed a cataract. $\mathrm{He}$ was treated with chemotherapy and also received $30 \mathrm{~Gy}$ to the orbit. Because of extensive orbital involvement no corneal shielding was used. The cataract developed two years after treatment and this was thought to be due to radiation rather than to the cortico-steroids used in the chemotherapy protocol.

\section{Discussion}

It has been shown ${ }^{2}$ that irradiation of the central zone of the lens, which has no mitotic activity, does not result in cataract formation. Cataracts form because of irradiation of the germinative zone of the anterior lens epi- 


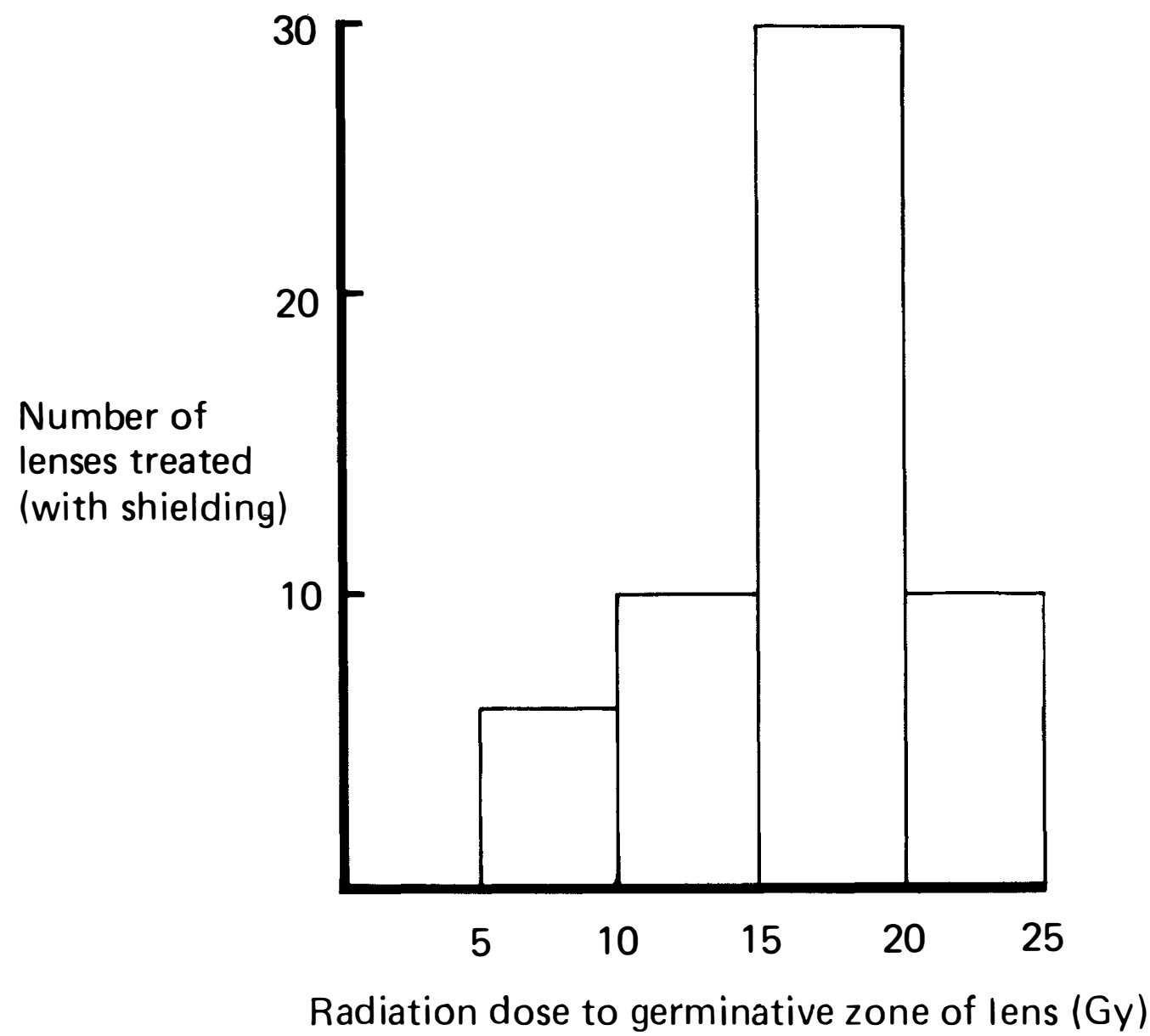

Fig. 2. Histogram of distribution of radiation dose to the germinative zone of the lens when anterior lens shielding is used. Five radiation induced cataracts were observed when lens shielding was used and these occurred at doses to the germinative zone of $15 \mathrm{~Gy}$ (3 lenses) and $18 \mathrm{~Gy}$ and $24 \mathrm{~Gy}$ (1 lens each).

thelium which is situated peripherally in the lens.

Radiation induced cataract has been extensively studied by Merriam and associates. ${ }^{1.2}$ Opacities appear centrally in the posterior subcapsular region as small granules or vacuoles forming a doughnut shape with a relatively clear central part. These changes may or may not progress sufficiently to interfere with vision. The probability of visual impairment, and the latent period between irradiation and development of the cataract, are both dependent on radiation dose. The higher the radiation dose the more probable is the progression to a mature cataract, and the shorter the latent period.
A high incidence of cataract from lens dosage above $40 \mathrm{~Gy}$ has been confirmed in several studies. Egbert et al. ${ }^{3}$ reported ocular complications in children with retinoblastoma who were treated with external beam radiotherapy; they received 50 to $60 \mathrm{~Gy}$ and had a minimum follow-up of two years. Seventy per cent of them developed cataract. Chan and Shukovsky ${ }^{5}$ found a high incidence of cataract in patients receiving $60 \mathrm{~Gy}$ to the lens during treatment for para-nasal sinus tumours. Parsons et al ${ }^{4}$ reviewed late ocular morbidity in 74 patients who received radiotherapy, mostly for para-nasal sinus or nasal cavity tumours, to a dose of 40 to $75 \mathrm{~Gy}$. All of these developed a cataract. No attempt was made to cal- 


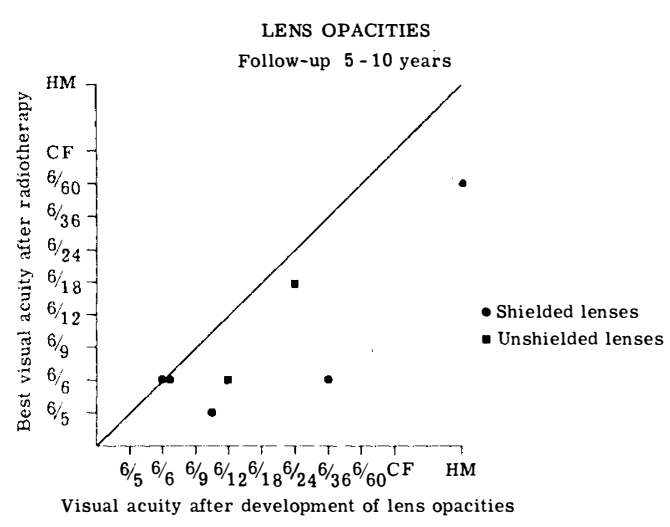

Fig. 3. Graph of reduction in visual acuity attributable to radiation induced cataract.

Circles $=$ shielded lenses

Squares $=$ unshielded lenses

In one case, where the lens received $30 \mathrm{~Gy}$, subsequent follow-up was at another centre. A radiation-induced cataract with slight reduction in vision was observed, but accurate visual acuity measurements were not obtained before death.

culate the lens dose in those who had lens shielding.

The incidence of cataract formation from lower doses is less clear. From our observation of a 64 per cent incidence of cataract by five years in patients receiving a lens dose of $30 \mathrm{~Gy}$, it is likely that almost all who receive this dose will ultimately develop cataract. The effect of doses below $20 \mathrm{~Gy}$ is especially controversial. Merriam and Focht ${ }^{1}$ estimated lens doses retrospectively in patients treated with $\mathrm{X}$-rays in the kilovoltage range. They found that the lowest dose to produce lens opacities was $2 \mathrm{~Gy}$ for a single exposure and $4 \mathrm{~Gy}$ for a fractionated course. They claimed that a lens dose greater than $10 \mathrm{~Gy}$ in a fractionated course always led ultimately to cataract formation with an average time of onset of 4 or 5 years. However, not all patients receiving lens irradiation during the period of the survey were examined, and the number of patients who received fractionated radiotherapy in each range of lens dose was small.

Deeg et al. ${ }^{9}$ studied the incidence of cataract formation in 277 patients undergoing bone marrow transplantation. These patients received whole body irradiation without lens shielding. After a single exposure of $10 \mathrm{~Gy} 70$ per cent developed a cataract within five years. The probability of cataract formation in those patients receiving a fractionated dose of 12 to 16 Gy and who had not received corticosteroids was 9 per cent at 5 years. This agrees with our figures of 12 per cent for lenses receiving a mean dose of $15 \mathrm{~Gy}$. It is possible that the risk of cataract formation from doses in this range has been previously overestimated.

\section{Disordered ocular lubrication}

Tears are composed of a superficial lipid layer, an aqueous middle layer, and a deep mucinous layer. The stability of the normal tear film is dependent on the presence of normal epithelial microvilli with adherent brush-like glycoproteins, which originate from sub-surface epithelial vesicles. Parsons et al. ${ }^{4}$ found that all patients receiving more than $50 \mathrm{~Gy}$ to the orbit developed a 'dry eye' but that the majority who received 30 to $40 \mathrm{~Gy}$ did not do so. Roth et al. ${ }^{10}$ in a study of the effects of fast neutrons on the eye found that fractionated doses of 220 to 860 neutron rad (approximately equivalent to 8 to $30 \mathrm{~Gy}$ of fractionated X-rays) led to tear-oil deficiency and corneal drying as a result of meibomian gland obliteration. Tear-film deficiency occurred mostly at doses equivalent to $40 \mathrm{~Gy}$ or greater of fractionated X-rays.

Our findings are broadly in agreement with these previous studies. No patient who

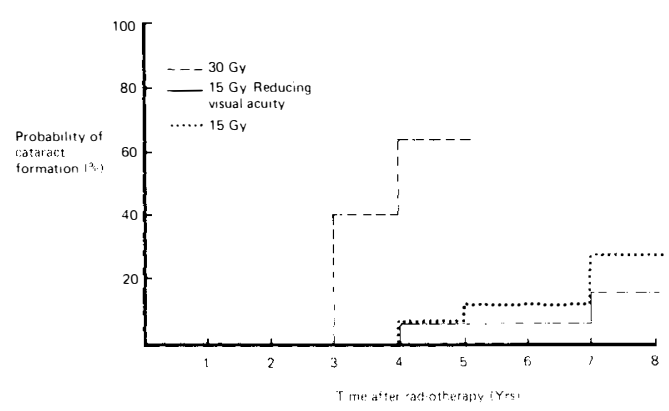

Fig. 4. Life-table estimate of probability of cataract formation at lens doses of $30 \mathrm{~Gy}$ and $15 \mathrm{~Gy}$ (mean)

Dose

$\begin{array}{llllllllll}\text { Years } & 1 & 2 & 3 & 4 & 5 & 6 & 7 & 8 & G y\end{array}$

$\begin{array}{llllllllllll}\text { No lenses } & 8 & 5 & 5 & 2.5 & 0.5 & - & - & - & 30\end{array}$ $\begin{array}{llllllllll}\text { at risk } & 56 & 44 & 36 & 28 & 19 & 15 & 12 & 6 & 15\end{array}$ 
Table III Incidence of late ocular morbidity. Incidence (per cent) of dry eye and corneal ulcer

\begin{tabular}{|c|c|c|c|c|}
\hline $\begin{array}{l}\text { No. of } \\
\text { patients }\end{array}$ & $\begin{array}{l}\text { No. of } \\
\text { orbits }\end{array}$ & $\begin{array}{c}\text { Orbital } \\
\text { dose }(G y)\end{array}$ & $\begin{array}{l}\text { No. of dry } \\
\text { eyes (\%) }\end{array}$ & $\begin{array}{c}\text { No. of } \\
\text { corneal } \\
\text { ulcers (\%) }\end{array}$ \\
\hline 17 & 21 & $6-29$ & $0(0)$ & $0(0)$ \\
\hline 43 & 44 & $30-39$ & $2(4.5)$ & $0(0)$ \\
\hline 13 & 13 & $40-49$ & $3(23)$ & $2(15)$ \\
\hline
\end{tabular}

received less than $30 \mathrm{~Gy}$ to the orbit developed disordered ocular lubrication and the incidence in those receiving 30 to $39 \mathrm{~Gy}$ was only 4.5 per cent (Table III). Inadequate ocular lubrication following radiotherapy may result from either radiation-induced ocular surface disease or radiation-induced lacrimal gland abnormalities or a combination of both. Corneal ulceration may'result from corneal drying, reduced sensation and radiationinduced ocular surface disease. Only two patients developed a small anterior stromal corneal ulcer which eventually healed. Both of these patients received over $40 \mathrm{~Gy}$ to the orbit.

\section{Retinopathy}

The effects of radiation on the retina have been described by several authors. ${ }^{3,4,11}$ The changes appear between 1.5 and 2 years after treatment. The main change is telangiectasia but capillary obstruction and microaneurysms also occur. Parsons et al. ${ }^{4}$ described severe radiation retinopathy in 12 of 13 patients receiving 50 to $70 \mathrm{~Gy}$ over 5 to 9 weeks.

At radiation doses of 50 to $60 \mathrm{~Gy}$ to the retina, fine and coarse pigment mottling with occasional microaneurysms occur. ${ }^{3} \mathrm{~A}$ dose of $80 \mathrm{~Gy}$ to the retina (which for example, may result from the application of 60 co plaques for the treatment of choroidal malignant melanoma) causes more severe retinal damage. Fluorescein leakage from telangiectatic vessels is seen at angiography and some patient have infarcts, haemorrhages and exudates. ${ }^{11}$ No retinopathy was observed in any of our patients. Of the 10 patients examined by fundus fluorescein angiography, who received a retinal dose of 25 to $30 \mathrm{~Gy}$, no retino-vascu- lar changes were detected. These results suggest that the risk of retinopathy with this dose of radiation is very small.

\section{Conclusions}

Orbital lymphoma can be successfully treated by moderate doses of radiotherapy (30 to $40 \mathrm{~Gy}$ ). In most cases the lens can be partially shielded from radiation and can receive 10 to $15 \mathrm{~Gy}$. At these dose levels early and late ocular morbidity caused by radiotherapy is slight, in contrast to the effects of higher doses of radiation ( 50 to $70 \mathrm{~Gy}$ ) which usually lead to disordered ocular lubrication, cataract and retinopathy.

\section{References}

${ }^{1}$ Merriam GR Jr and Focht EF: A clinical study of radiation cataracts and the relationship to dose. Am. J. Roentgen. 1957, 77: 759-85.

2 Merriam GR Jr, and Worgul BV: Experimental radiation cataract-its clinical relevance. Bull. N.Y. Acad. Med. 1983, 59: 372-92.

${ }^{3}$ Egbert PR, Donaldson SS, Moazed K and Rosenthal AR: Visual results and ocular complications following radiotherapy for retinoblastoma. Arch. Ophthalmol. 1978, 96: 1826-30.

${ }^{4}$ Parsons JT, Fitzgerald CR, Hood CI, Ellingwood $\mathrm{KE}$, Bova FJ and Million RR: The effects of irradiation on the eye and optic nerve. Int. J. Radiat. Oncol. Biol. Phys. 1983, 9: 609-22.

${ }^{5}$ Chan RC and Shukovsky LJ: Effects of irradiation on the eye. Radiology 1976, 120: 673-5.

${ }^{6}$ Carbone PP, Kaplan HS, Musshoff K, Smithers DW and Tubiana M: Report of the committee on Hodgkin's disease staging classification. Cancer Res. 1971, 31: 1860-1.

${ }^{7}$ Morgan $\mathrm{G}$ and Harry J: Lymphocytic tumours of indeterminate nature: a 5-year follow-up of 98 conjunctival and orbital lesions. $B r . J$. Ophthalmol. 1978, 62: 381-3.

${ }^{8}$ Bessell EM, Henk JM, Whitelocke RAF and Wright JE: Primary non-Hodgkin's lymphoma of the orbit. Radiotherapy and Oncology 1987 (submitted).

${ }^{9}$ Deeg HJ, Flournoy N, Sullivan KM, Sheehan K, Buckner CD, Sanders JE, Storb R, Witherspoon RP and Thomas ED: Cataracts after total body irradiation and marrow transplantation: a sparing effect of dose fractionation. Int. J. Radiat. Oncol. Biol. Phys. 1984, 10: 957-64.

${ }^{10}$ Roth J, Brown N, Catterall M and Beal A: Effects of fast neutrons on the eye. Br. J. Ophthalmol. 1976, 60: 236-44.

${ }^{11}$ Hayreh SS: Post-radiation retinopathy-a fluorescein fundus angiographic study. $\mathrm{Br} . \quad \mathrm{J}$. Ophthalmol. 1970, 54: 705-14. 